\title{
Role of surgical simulation to enhance the knowledge of surgical procedures
}

\author{
Deepak Raj Singh', Sujan Regmee², Rupesh Chakradhar², Aditi Agrawal², Kshitiz Bhujel², \\ Susant Gurung ${ }^{2}$, Uttam Laudari ${ }^{3}$, Tanka Prasad Bohara ${ }^{2}$
}

\section{Abstract}

Introduction: Surgical simulator is a computer based technology used to educate and train medical students and practitioners without the need for a patient, cadaver or an animal model. Touch surgery ${ }^{\mathrm{TM}}(\mathrm{TS})$ is a free of cost smartphone based application used for teaching surgical procedures. The participants in this study were specifically assessed on the increase of their knowledge in the key steps of laparoscopic cholecystectomy after practicing on the smartphone simulator, TS.

Methods: A non-randomized controlled trial (uncontrolled longitudinal study) was carried out among 20 final year medical students. Their knowledge on surgical procedure was assessed through a questionnaire prepared regarding the steps of laparoscopic cholecystectomy before and after exposure to TS. Study Variable was the scores to a questionnaire before and after exposure to the surgical simulation.

Results: Mean pre-practice score was $25.85 \pm 11.05$ and mean post practice score was $80 \pm 10.62(\mathrm{p}<0.001)$.

Conclusion: This study suggests that the use of TS can increase the knowledge of the students about laparoscopic cholecystectomy. This can definitely be used for teaching students, other surgical procedures.

Keywords: Surgical simulation; Simulate laparoscopy cholecystectomy; Touch Surgery.

\section{Author affiliations:}

1 Department of Surgery, KIST Medical College, Lalitpur, Nepal.

2 Department of Surgery, Kathmandu Medical College, Kathmandu, Nepal.

${ }^{3}$ Department of Surgery, Hospital for Advanced Medicine and Surgery, Kathmandu, Nepal.

\section{Correspondence:}

Dr. Deepak Raj Singh,

Department of surgery, KIST Medical College \& Teaching Hospital,

Lalitpur, Nepal

Email: drsinghnp@gmail.com

ORCID:https://orcid.org/0000-0001-81749619

Disclosures:

Ethical Clearance: IRB of KMCTH

Conflict of interest: None

Financial aid: None

Copyright information:

Authors retain copyright and grant the journal right of first publication with the work simultaneously licensed under Creative Commons Attribution License under CC-BY 4.0 that allows others to share the work with an acknowledgement of the works's authorship and initial publication of this journal.

\section{How to cite this article:}

Singh DR, Regmee S, Chakradhar R, Agarwal A, Bhujel K, Gurung S et al. Role of surgical simulation to enhance the knowledge of surgical procedures. J Soc Surg Nep. 2020 Dec;23(2);14-17.

DOI:

https://doi.org/10.3126/jssn.v23i2.35798 


\section{Introduction}

Simulation is an educational technique that allows the interactive performance of the trainee in an environment that recreates or replicates a real-world scenario, but is not identical to real life. ${ }^{1}$ Simulation can include anything from the use of standardized models and less reliable bench models, to highly reliable technologically advanced virtual reality (VR) systems. Simulation has been used and is currently well established in several industries including the military, aviation, and astronautics. ${ }^{2,3}$ In the aviation industry, pilots must undergo exhaustive drills and certification on flight simulation before being allowed to fly a plane. The first Boeing 777 flight was flown with over $1000 \mathrm{hrs}$ of simulation training. 4 Though slow but the use of simulation in surgical training is gaining significant momentum.

A surgical simulator is a computer based technology developed to simulate surgical procedures to train medical professionals without the need of a patient, cadaver or animal before working with the live patients. It is a method of training minimally-invasive operative techniques, similar to simulator training of airline pilots, which helps learners to deal with the details of a surgical procedure without hampering a patient's safety. Surgeons have been taught in the past centuries through the Halstedian model, which involved learning the craft through training. ${ }^{5}$ Though effective, there are many factors that suggests a need for reconsideration in the instructional strategy of surgical education. ${ }^{2}$ They are useful for development of an ability to perform three dimensional actions using a two dimensional screen as a guide. Simulation has now become a key part of providing this objective training and assessment allowing mistakes to be made in a safe environment, and respect the human ethics that exists outside the realm of pure technical ability. ${ }^{6,7}$ Moreover it saves money as in an report form Bridges et al from USA it was found that the increase in operative time ascribed to resident training translated into a cost of $\$ 53$ million a year. ${ }^{8}$

Touch surgery (TS) a novel, innovative and cost free application for mobile devices teaches different surgical procedure, offers both training and self-assessments for its users. ${ }^{9}$ It can be used within the constraints of work hour restriction and limited clinical exposures. ${ }^{6}$

This study was carried out with the objective to assess the role of simulation in enhancing the knowledge of certain surgical procedure. It was done to assess the effectiveness of smartphone based simulation in the process of learning procedures particularly in the field of surgery. It is also important to document the merits and the demerits of simulation education, since it is a novel technique and a concept that is growing rapidly. The participants were specifically assessed on the increase of their knowledge in the key steps of laparoscopic cholecystectomy after practicing on the smartphone simulator, TS.

\section{Methods}

A non-randomized controlled trial (uncontrolled longitudinal study) was carried out at Kathmandu Medical College teaching hospital from 1st Nov to 1st Dec 2017 among the final year medical students. Their surgical procedure knowledge was assessed through a questionnaire prepared regarding the steps of laparoscopic cholecystectomy before and after exposure to the smartphone based surgical simulator. This study was done to assess the role of surgical simulation to increase the knowledge of surgical procedures.

Non Probability Convenience sampling method was applied. It was conducted as a pilot study including 20 students only, as the number of students posted in surgery department during the study period were 20 . The confidence interval was expected to be narrow since simulations have more benefits than risks from learning point of view. Final year medical student who had not used the proposed application before the pretest was the criteria for sample selection.

Study Variable was the scores to a questionnaire before and after exposure to the surgical simulation.

Pre-test data collection was done through the preset questionnaire among the participants who were then introduced to TS. Participants were allowed to practice the procedure of Laparoscopic cholecystectomy in the mobile application which is an android and IOS based application that is free to download and use. It has simulations of Laparoscopic cholecystectomy, taught in step by step manner to the users. Once the maximum score attained, the participant's knowledge on the procedure was again assessed by the preset posttest questionnaire.

Pretest and post test scores compared using paired t test. SPSS 20 is used to analyze the data. P value $<0.05$ was considered significant. Ethical approval was taken from Institutional Review Board. Permission was taken from Touch Surgery for the use of the application in the study.

\section{Results}

Twenty students were enrolled in the study. Among them, 08 were girls (40\%) and 12 were boys (60\%). Mean prepractice score was $25.85 \pm 11.05$ and mean post practice score was $80 \pm 10.62(\mathrm{p}<0.001)$.

\section{Discussion}

Surgical simulation are of various types, there are simulation models which gives the hands on practice feature, like the surgical simulators for laparoscopic and robotic surgeries. On the contrary Touch Surgery is a simple smart phone based application that helps to learn and rehearse surgical steps for various procedures. The positive role of simulation via Touch surgery for enhancement of knowledge has been seen to be highly realistic and useful. Kowalewski K.F et in his study published in 2017 regarding the validation 
of Touch Surgery for cognitive training and assessment of laparoscopic cholecystectomy compared its use in 54 surgeons and 51 medical students to complete the validation study. He found that surgeons outperformed students with TS in patient preparation (students $=45.0 \pm 19.1 \%$; surgeons $=57.3 \pm 15.2 \% ; \mathrm{p}<0.0010$, access and laparoscopy (students $=70.2 \pm 10.9 \% ; \quad$ surgeons $=75.9 \pm 9.7 \% ; \quad \mathrm{p}=0.008$ ) and LC (students $=69.8 \pm 12.4 \%$; surgeons $=77.7 \pm 9.6 \%$; $\mathrm{p}<0.001)$. This showed that both groups agreed that TS was a highly useful and realistic application with the conclusion that TS is an acceptable application for learning cognitive aspects of LC with established concept, face, and content validity. ${ }^{9}$

Role of simulation has also been validated in orthopedic surgical training. Sugand K and his collegues in 2015 made a publication in which 39 novices and 10 experts were recruited to complete four simulation modules on surgical decision making that represented the procedural steps of intramedullary nailing and found that graphics, simulated environment and the procedural steps were realistic. They concluded that the application was useful for surgical training and rehearsal and further commented that it should be implemented within the curriculum and would want to use it to learn other surgical procedures..$^{10,11}$

In our study the method was different, where the pre and post-practice scores of the same individual was compared. This method of comparison of a pre and post-practice score following a training module is a known method of accessing the utility of the particular testing module. This is often

Table 1. Pre-test and Post test scores

\begin{tabular}{|c|c|c|}
\hline S. No & Pre Test Scores & Post Test Scores \\
\hline 1. & 16 & 30 \\
\hline 2. & 12 & 36 \\
\hline 3. & 3 & 32 \\
\hline 4. & 6 & 32 \\
\hline 5. & 7 & 35 \\
\hline 6. & 6 & 30 \\
\hline 7. & 9 & 29 \\
\hline 8. & 5 & 18 \\
\hline 9. & 10 & 33 \\
\hline 10. & 6 & 28 \\
\hline 11. & 13 & 32 \\
\hline 12. & 9 & 33 \\
\hline 13. & 16 & 34 \\
\hline 14. & 16 & 32 \\
\hline 15. & 7 & 34 \\
\hline 16. & 13 & 26 \\
\hline 17. & 16 & 35 \\
\hline 18. & 16 & 28 \\
\hline 19. & 9 & 35 \\
\hline 20. & 7 & 32 \\
\hline
\end{tabular}

implemented in trainings and workshops for accessing the knowledge gained by the participant in the training. The mean pre-practice score was $25.85 \pm 11.05$ and mean post practice score was $80 \pm 10.62(\mathrm{p}<0.001)$. This result implies that the student who used Touch Surgery for practicing laparoscopic cholecystectomy had a significant increase in the scores obtained in the questionnaire after practicing the surgery using the application. What we cannot imply from the result is the fact that the participant can directly reproduce the skills in a real time scenario that is at the operating theatre. But the participant will definitely have an edge on understanding the procedure after the training session. This will help junior medical school student to rehearse the steps of the given surgery using TS, so when they get an actual opportunity to see the surgery being done they can understand the procedure well. ${ }^{12}$ As far as the surgical trainees are concerned this can help them assist the surgery better if they have rehearsed the surgery using the application. Surgery is a team work, and a team is only as strong as its weakest link. A novice surgical trainee who happens to assist cases at the theatre but has not seen the surgery at all or as frequently as required can welcome complications in the surgery, the results of which can be catastrophic at times.

Using TS is just like playing a video game; it gives enjoyment to the users and motivates them to continue its use. There is no need of supervisors and access to training centers, also there is no time restrictions for its use, which is a reason for its utility and popularity. ${ }^{9}$

One of the most underrated and unidentified aspect of surgical training is the training of the scrub nurse and other nursing staff. In a world where we expect surgeons to train for over six years to get a sub specialization degree, nursing staffs who scrubs with the surgeon is hardly trained about the procedures being undertaken. Here comes the role of simulation which aid in preparation of the nursing students for their management assessment and the transition to a qualified nurse. Simulation is perceived by the nursing students not only as an effective learning mechanism, but is also reported to be enjoyable to the extent that respondents appeal for its inclusion within the undergraduate programme to be increased and commenced earlier in the course. ${ }^{13}$ The use of TS in such nursing staff can help them learn and revise the steps of surgery thus making the surgical procedure smooth and uneventful. ${ }^{14}$

Moreover for the senior consultants and surgeons, TS can be an aid to rehearse the steps of a particular surgery that is rare or is being done after a long time gap. This helps them revise the surgical steps quickly so that they can focus on other aspect of patient care in the given time. ${ }^{9,15}$

Limitations: The results obtained after the intervention may not be solely due to it. Potential biases can be on selection, process and results. This means that after a pre- test, the participants will be practicing the surgical simulation without being monitored strictly. They might enhance their 
knowledge through other means too for instance by reading more extensively about the topic.

\section{Conclusion}

This study suggests that the use of TS can increase the knowledge of the students about laparoscopic cholecystectomy. This can definitely be used for teaching students, other surgical procedures.

\section{References}

1. Issenberg SB, McGaghie WC, Hart IR et al. Simulation technology for health care professional skills training and assessment. JAMA. 1999;282(9):861-6.

2. Cosman P, Cartmill J. Simulation in Surgical Education: "Nintendoplasty." 2011;1(212):156-65.

3. Scott DJ, Cendan JC, Pugh CM, Minter RM, Dunnington GL K, RA. The changing face of surgical education: simulation as the new paradigm. J Surg Res. 2008;147:189-93.

4. Satava RM. Accomplishments and challenges of surgical simulation: Dawning of the next-generation surgical education. Surg Endosc. 2001;15(3):23241.

5. Kerr B, Leary JPO. The training of the surgeon: Dr. Halsted's greatest legacy. Am Surg.1999 Nov;65(11):1101-2.

6. Agha RA, Fowler AJ. The validity of surgical simulation. Can J Surg. 2014;57(4):226-7.

7. Hamdorf JM HJ. Acquiring surgical skills. Br J Surg. 2000;87:28-37.

8. Bridges M DD. The financial impact of teaching surgical residents in the operating room. Am J Surg. 1999;177(1):28-32.

9. Kowalewski KF, Hendrie JD, Schmidt MW, Proctor
T, Paul S, Garrow CR, et al. Validation of the mobile serious game application Touch Surgery ${ }^{\mathrm{TM}}$ for cognitive training and assessment of laparoscopic cholecystectomy. Surg Endosc. 2017;31(10):405866.

10. Sugand K, Mawkin M, Gupte C. Validating Touch Surgery $^{\mathrm{TM}}$ : A cognitive task simulation and rehearsal app for intramedullary femoral nailing. Int J care Inj. 2015;46(11):2212-6.

11. Paro JAM, Luan A, Lee GK. Validation of a Cognitive Task Simulation and Rehearsal tool for Open carpal tunnel release. Arch Plast Surg. 2017;44:223-7.

12. Antonoff MB, Shelstad RC, Schmitz C, Chipman J, D'Cunha J. A Novel Critical Skills Curriculum for Surgical Interns Incorporating Simulation Training Improves Readiness for Acute Inpatient Care. J Surg Educ. 2009;66(5):248-54.

13. McCaughey CS, Traynor MK. The role of simulation in nurse education. Nurse Educ Today. 2010;30(8):827-32.

14. Fort C, Fitzgerald B. How simulation improves perioperative nursing. OR Nurse. 2011;5(2):36-42.

15. Tan SSY, Sarker SK. Simulation in surgery: A review. Scott Med J. 2011;56(2):104-9. 